\title{
Time-Restricted Eating in Women - A Pilot Study
}

Siobhan T. Smith ${ }^{1}$, Jordan C. LeSarge ${ }^{1}$, Peter W. R. Lemon ${ }^{1}$

'University of Western Ontario

BACKGROUND: There is a growing interest in intermittent fasting as a method of body fat loss. However, research on time-restricted eating, a type of intermittent fasting, is more limited. The purpose of this study was to assess the effects of time-restricted eating on fat mass in women.

METHODS: 20 healthy, young (21.3 years \pm 1.2 years), body mass stable ( $\pm 2.27 \mathrm{~kg}$ in the past 6 months) women completed a 4week time-restricted eating study. During the intervention, participants restricted their daily food intake to an 8-hour period, i.e., between 1200 to 2000 hours. Changes in fat mass and body mass were assessed via densitometry (Bod Pod ${ }^{\circledR}$ ). Hunger, satisfaction, fullness, and adherence were assessed on a weekly basis by self-report.

RESULTS: A $0.6 \mathrm{~kg} \pm 1 \mathrm{~kg}$ decrease in body mass occurred after the 4 weeks $(p=0.015, n=20)$ but changes in fat mass were nonsignificant. Women who performed strength training during the study experienced a $0.7 \mathrm{~kg} \pm 0.5 \mathrm{~kg}$ decrease in fat mass $(p=0.037$, $\mathrm{n}=5$ ). Overall protocol adherence was high $(>5.5 \mathrm{~d} / \mathrm{wk})$ throughout the intervention. Hunger, satisfaction, and fullness remained constant over the study.

\section{Background}

Obesity has become a significant public health problem in Canada that is only getting worse [1]. Obesity is a health risk for mortality, hypertension, dyslipidemia, type 2 diabetes, coronary heart disease, strokes, osteoarthritis, sleep apnea, cancer, mental illness, low quality of life, and more [2]. In 2014, $20.2 \%$ of Canadians aged 18 and older, roughly 5.3 million adults, were obese and $33.8 \%$ overweight [3]. For years, lifestyle interventions, including reduced energy intake and increased regular exercise, have been the first-line therapy in efforts to combat obesity. However, these lifestyle changes require substantial attention to nutrient quality and quantity of food consumed as well as physical activity. Compliance to this type of lifestyle intervention is often poor and successful long-term fat loss has been limited to a small percentage of individuals [4]. Hence, novel interventions are urgently needed. Time-restricted eating appears to be a promising solution.

Intermittent fasting is a term covering several sub-categories of low energy dieting including: alternate day fasting, whole day fasting, and time-restricted eating/feeding. A recent review of intermittent fasting found that alternate day fasting and whole day fasting approaches produced significant reductions in body fat; however, the same review noted that research on time-restricted eating is more limited, and clear conclusions cannot be made at the present time [5].

Time-restricted eating is defined as ad libitum (unrestricted) energy intake within a controlled time frame, often a 3-12 hour time period daily. Recently, in 12-week-old male mice, an 8hour time-restricted feeding pattern was compared to 24 hour per day eating over more than 100 days [6]. Although both groups consumed equal daily quantities of the same food, the ad libitum animals became obese and developed several negative health biomarkers while the mice in the timerestricted group were protected against both obesity and the adverse health consequences [6].

Furthermore, the time-restricted animals maintained a lower respiratory exchange ratio (RER) throughout the day and a greater daily oxygen consumption suggesting an up-regulated fat metabolism [6].

Time-restricted eating has ample potential for fat mass loss because of what appears to be an associated up-regulation of fat metabolism. In comparison to maintaining an energy deficit chronically, this approach represents a lifestyle that is likely more sustainable, i.e., it can be accomplished by simply skipping breakfast and may promote fat loss without any energy restriction (i.e. diet quality and quantity). Further, a daily energy intake decrease could result, due to the smaller window of eating. If so, this could produce additional 
fat and body mass losses. At least anecdotally, it is a sustainable diet intervention for body fat mass loss and, as a result, could lead to a number of health benefits.

The purpose of this study was to assess the effects of 8-hour eating and 16-hour fasting periods daily, irrespective of diet quality or quantity, over 4weeks on both body mass and body fat mass in young healthy women. The hypothesis was that the time-restricted eating would produce both body mass and fat loss. A secondary hypothesis was that adherence, hunger, satisfaction, and fullness would remain steady over the four weeks.

\section{Methods \\ Participants}

Twenty-four women (age $=21.3$ years \pm 1.2 years) were recruited. Four participants dropped out before completing the study $(n=20)$. All women self-reported a stable body mass $( \pm 2.27 \mathrm{~kg})$ over the past 6 months. Diabetic and/or pregnant individuals or those with a history of fainting, low blood sugar, heart disease, migraines, or heartburn were excluded. In addition, participants less than 18 years of age, as well as those with health or exercise performance reasons that precluded skipping breakfast (assessed by self-report) were excluded from the study. The remaining participants were informed of the purpose of the study and gave their written consent prior to any testing. The project was approved by the Western University Health Science Research Ethics Board.

\section{Intervention}

Participants completed a 4-week intervention where they restricted their daily food intake to an 8-hour period, i.e., between 1200 and 2000 hours. During the remaining 16-hour fasting period (2000 to 1200 hours), all of the participants were encouraged to drink zero calorie beverages (diet soda pop, black coffee, tea, water, etc.) to maintain normal hydration. There was no attempt to restrict food quality or quantity during the feeding period (1200 to 2000 hours). The only restriction was the time period each day when food was consumed. Participants were not asked to keep a food diary. Participants were also asked to keep their exercise type and frequency constant. At the end of the intervention participants were asked retrospectively what exercise they did in a typical week during the intervention.

\section{Body Mass and Composition}

Participants' body mass, fat mass, and fat free mass were monitored non-invasively using a weigh scale and densitometry via air displacement $\left(\right.$ Bod Pod $^{\circledR}$ ) at the same time of day prior to and after the 4-week dietary intervention. Participants wore spandex exercise clothing, no jewelry, and a Lycra $^{\circledR}$ cap for both measures. They were also asked to refrain from exercising, eating, or drinking liquids two hours before the measurement time. The Bod Pod $^{\circledR}$ measures body volume via air displacement and together with an accurate measure of body mass enables body density to be calculated (density = mass/volume). Then, percent fat (percent of body mass that is fat mass) is estimated with the Siri equation, using tissue densities from human cadaver analysis [7].

\section{Adherence}

Adherence, the number of days the diet was followed, was assessed on a weekly basis throughout the 4-weeks by self-report. Participants were asked to record the number of days in the past week they had complied with the 8-hour feeding regime. Participants were sent weekly email reminders to record their adherence. The results for the 4-weeks were collected at the end of the intervention.

\section{Hunger, Satisfaction, and Fullness}

Hunger, satisfaction, and fullness with the time restricted eating protocol were monitored on a weekly basis using a validated visual analog scale (VVAS; Figure 1) [8]. Briefly, this consists of a 100 $\mathrm{mm}$ line where participants marked a value on the line for each of the categories, which represented how they felt in that category over the past week. For hunger, 0 represented "Not hungry at all" and 100 represented "Never been more hungry"; for satisfaction, 0 represented "Completely empty" and 100 represented "Cannot eat another bite"; and for fullness, 0 represented "Not full at all" and 100 represented "Totally full". Participants filled out the VVAS at the same time each week and responses were quantified using a ruler to the nearest $\mathrm{mm}$. Participants were sent weekly emails reminding them to record their hunger, satisfaction, and fullness scores. The results for the 4-weeks were collected at the end of the intervention.

\section{Statistics}

Statistics were completed using SPSS (Statistical Package for the Social Science) software 2016 version 24 . Fat mass change and body mass change data were assessed for significance using a two-tailed paired t-test. *Significance was set at $P<0.05$. Adherence and hunger, satisfaction, and fullness were assessed with repeated-measures one-way ANOVA (treatment $X$ time) procedures and post hoc testing (Tukey) was done, where 
appropriate. Data were presented as mean \pm standard deviation (SD).

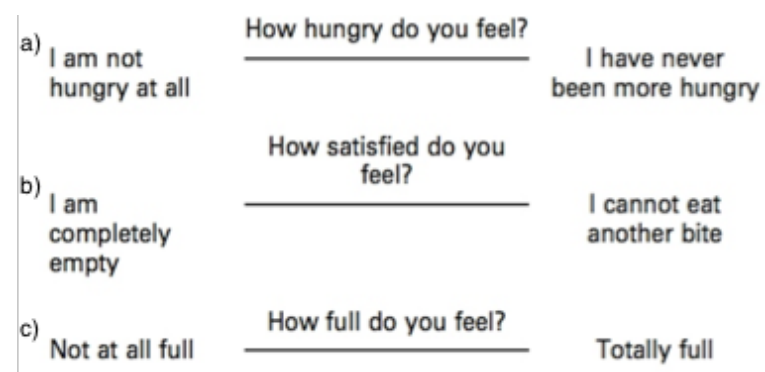

Figure 1 - Validated Visual Analog Scale for hunger (a), satisfaction (b), and fullness (c).

\section{Results}

\section{Body Mass}

There was a significant $(p=0.015)$ decrease of $0.6 \mathrm{~kg}+1 \mathrm{~kg}$ in body mass for the group following the 4 -week time-restricted feeding intervention (Table 1).

Table 1 - Participants' body mass $(\mathrm{kg})$ change following a 4-week time-restricted eating program in young, healthy, women.

\begin{tabular}{|c|c|c|c|}
\hline $\begin{array}{l}\text { Participant } \\
\text { Number }\end{array}$ & $\begin{array}{l}\text { Body Mass at } 0 \\
\mathrm{wk}(\mathrm{kg})\end{array}$ & $\begin{array}{l}\text { Body Mass at } 4 \\
\mathrm{wk}(\mathrm{kg})\end{array}$ & $\begin{array}{l}\text { Change in Body Mass (kg) } \\
\text { (Final - Initial) }\end{array}$ \\
\hline 1 & 66.4 & 65.5 & -0.9 \\
\hline 2 & 62.2 & 61.9 & -0.3 \\
\hline 3 & 71.9 & 72.9 & 1.0 \\
\hline 4 & 54.8 & 53.7 & -1.1 \\
\hline 5 & 55.1 & 54.1 & -1.0 \\
\hline 6 & 52.6 & 53.5 & 0.9 \\
\hline 7 & 56.8 & 57.8 & 1.0 \\
\hline 8 & 88.5 & 87.2 & -1.3 \\
\hline 9 & 63.7 & 64.7 & 1.0 \\
\hline 10 & 47.7 & 47.1 & -0.6 \\
\hline 11 & 59.1 & 58.0 & -1.1 \\
\hline 12 & 54.1 & 53.2 & -0.9 \\
\hline 13 & 94.8 & 93.4 & -1.4 \\
\hline 15 & 66.6 & 65.5 & -1.1 \\
\hline 17 & 86.2 & 83.5 & -2.7 \\
\hline 18 & 66.3 & 65.1 & -1.2 \\
\hline 19 & 64.4 & 62.8 & -1.6 \\
\hline 20 & 77.8 & 78.5 & 0.7 \\
\hline 21 & 56.9 & 55.5 & -1.4 \\
\hline 22 & 56.4 & 56.0 & -0.4 \\
\hline
\end{tabular}

\begin{tabular}{llll} 
Mean & 65.1 & 64.5 & -0.6 \\
SD & 12.5 & 12.3 & 1.0 \\
p-value & - & - & 0.015 \\
\hline
\end{tabular}

Fat Mass

There was no significant $(p=0.948)$ decrease in fat mass after the 4-week timerestricted feeding intervention for the group (Table 2); however, participant 12 lost $3.2 \mathrm{~kg}$ of fat and participants $5,7,13,19$, and 21 lost more than $1 \mathrm{~kg}$ of fat.

Table 2 - Participants' fat mass (kg) change following a 4-week time-restricted eating program in young, healthy, women.

\begin{tabular}{|c|c|c|c|}
\hline $\begin{array}{l}\text { Participant } \\
\text { Number }\end{array}$ & $\begin{array}{l}\text { Fat Mass at } 0 \\
\text { wk (kg) }\end{array}$ & $\begin{array}{l}\text { Fat Mass at } 4 \\
\text { wk (kg) }\end{array}$ & $\begin{array}{l}\text { Change in Fat Mass }(\mathrm{kg}) \\
\text { (Final-Initial) }\end{array}$ \\
\hline 1 & 15.1 & 14.9 & -0.2 \\
\hline 2 & 21.0 & 20.5 & -0.5 \\
\hline 3 & 16.6 & 16.1 & -0.5 \\
\hline 4 & 10.2 & 10.2 & 0.0 \\
\hline 5 & 9.5 & 8.3 & -1.2 \\
\hline 6 & 11.0 & 11.4 & 0.4 \\
\hline 7 & 14.9 & 13.9 & -1.0 \\
\hline 8 & 31.4 & 31.3 & -0.1 \\
\hline 9 & 14.4 & 16.2 & 1.8 \\
\hline 10 & 9.9 & 9.3 & -0.6 \\
\hline 11 & 12.1 & 12.4 & 0.3 \\
\hline 12 & 16.7 & 13.5 & -3.2 \\
\hline 13 & 38.5 & 37.5 & -1.0 \\
\hline 15 & 16.7 & 17.6 & 0.9 \\
\hline 17 & 34.7 & 37.0 & 2.3 \\
\hline 18 & 16.1 & 18.4 & 2.3 \\
\hline 19 & 18.3 & 17.1 & -1.2 \\
\hline 20 & 22.3 & 23.9 & 1.6 \\
\hline 21 & 11.2 & 10.0 & -1.2 \\
\hline 22 & 13.5 & 14.2 & 0.7 \\
\hline Mean & 17.7 & 17.7 & 0.0 \\
\hline SD & 8.0 & 8.4 & 1.3 \\
\hline p-value & - & - & 0.948 \\
\hline
\end{tabular}

Exercise Effect

Participants $(n=5)$ that strength trained $(\geq 3$ $d / w k)$ had a significant $(p=0.037)$ decrease in fat mass of $0.7 \mathrm{~kg} \pm 0.5 \mathrm{~kg}$ following the 4 -week 
intervention (Table 3 ) while still reaching an acceptable level of power (Power $>0.8$ ).

Table 3 - Participants' fat mass $(\mathrm{kg})$ change following a 4-week time-restricted eating diet in young, healthy, women. Individuals are split into groups based on the type and frequency of their normal exercise.

\begin{tabular}{|c|c|c|c|c|}
\hline Participant Number & $\begin{array}{l}\text { Non- } \\
\text { exercisers } \\
\text { (Group 1) }\end{array}$ & $\begin{array}{l}\text { Endurance } \\
\text { exercisers } \\
\text { (Group 2) }\end{array}$ & $\begin{array}{l}\text { Resistance } \\
\text { exercisers } \\
\text { (Group 3) }\end{array}$ & $\begin{array}{l}\text { Endurance- } \\
\text { resistance } \\
\text { exercisers } \\
\text { (Group 4) }\end{array}$ \\
\hline 1 & & & & -0.2 \\
\hline 2 & -0.5 & & & \\
\hline 3 & & & -0.5 & \\
\hline 4 & & & 0 & \\
\hline 5 & & & -1.2 & \\
\hline 6 & & 0.4 & & \\
\hline 7 & -1 & & & \\
\hline 8 & & -0.1 & & \\
\hline 9 & 1.8 & & & \\
\hline 10 & & & -0.6 & \\
\hline 11 & & & & 0.3 \\
\hline 12 & & & & -3.2 \\
\hline 13 & -1 & & & \\
\hline 15 & & & & 0.9 \\
\hline 17 & 2.3 & & & \\
\hline 18 & & & & 2.3 \\
\hline 19 & -1.2 & & & \\
\hline 20 & & & & 1.6 \\
\hline 21 & & & -1.2 & \\
\hline 22 & & & & 0.7 \\
\hline Mean & 0.1 & 0.2 & -0.7 & 0.3 \\
\hline SD & 1.4 & 0.3 & 0.5 & 1.6 \\
\hline p-value & 0.921 & 0.656 & 0.037 & 0.626 \\
\hline
\end{tabular}

\section{Adherence}

Self-reported adherence remained high during the 4 weeks (Figure 2a) averaging greater than 5.5 days per week (d/wk). There was no significant difference in adherence among the weeks $(p=0.902)$.

\section{Hunger, Satisfaction, and Fullness}

Self-reported hunger $(p=0.877)$, satisfaction $(p=0.589)$, and fullness $(p=0.812)$ from the VVAS did not change over the 4 weeks. Hunger averaged $45 \mathrm{~mm}$ (Figure $2 \mathrm{~b}$ ), satisfaction averaged $51 \mathrm{~mm}$ (Figure 2c), and fullness averaged $51 \mathrm{~mm}$ (Figure 2d) over the 4-weeks.
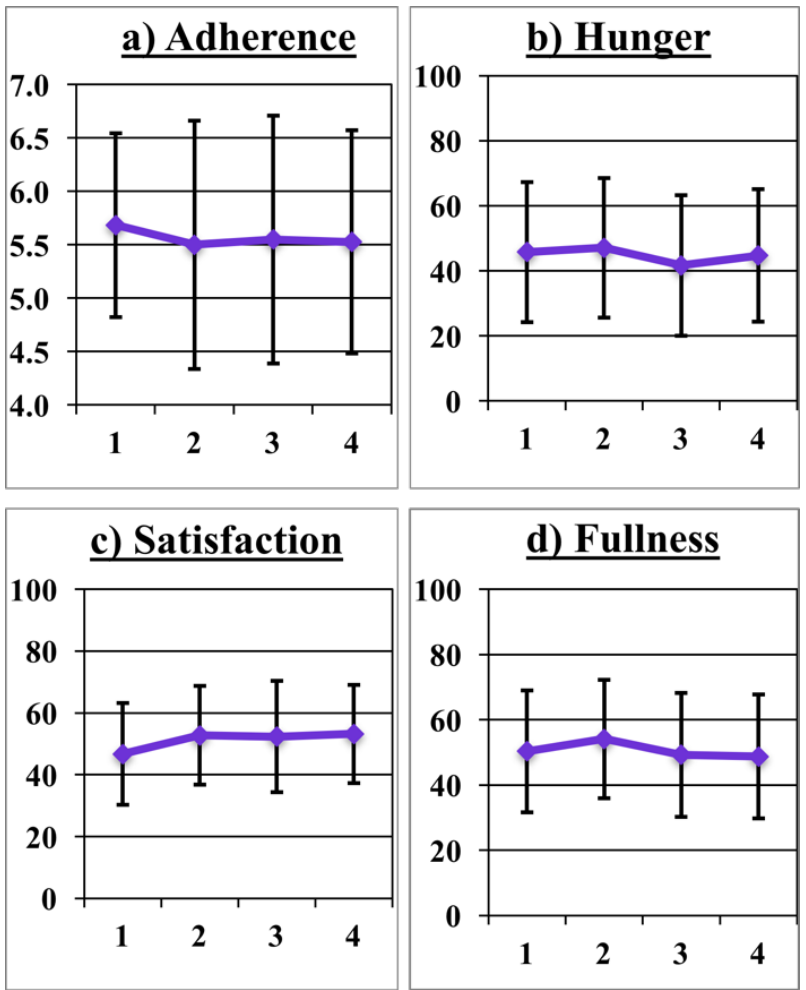

Figure 2 - Average adherence $(p=0.902)(d / w k$.$) ,$ hunger $(p=0.877)$, satisfaction $(p=0.589)$, and fullness $(p=0.812)$ from VVAS $(\mathrm{mm})$ to a 4-week time-restricted eating program in young, healthy women. Values are expressed as mean \pm SD.

\section{Discussion}

The pre-post values indicated a significant decrease in body mass of $0.6 \mathrm{~kg} \pm 1 \mathrm{~kg}$ (Table 1) with no significant change in fat mass (Table 2) following the 4-week time-restricted feeding intervention. Unlike the study in male mice by Hatori et al [6] and our hypothesis, it does not appear that time-restricted eating produces fat loss over a 4week period in women. Typically, alternate day fasting results in a net energy intake decrease and fat loss but these studies are usually 10-weeks in duration [9]. Although unmeasured in this study, this may not have been the case here or perhaps 4weeks is an insufficient duration to obtain significant results. If so, this could explain the greater fat loss observed with the alternate day fasting approach [9]. Of course, fat mass is the more important measure because the loss in body mass could have been due to water, muscle, fat, or a combination of 
all three. In our study, there was considerable variability in fat mass loss among participants (Table 2). The time-restricted eating was clearly successful for several individuals, including participant 12 who lost $3.2 \mathrm{~kg}$ of fat mass in just 4weeks, while clearly unsuccessful for others. This may be due to increases in energy intake over the study. Although energy intake was not measured, several individuals indicated that they felt they ate more during the intervention than they had prior to the study because they worried about feeling hungry during the fasting period. If so, this could explain the fat mass gain that some experienced. In the study by Hatori et al [6] with mice, diet composition was controlled and both the 24-hour eating and 8-hour eating groups ate the same amount of food. In free-living humans, diet is much more difficult to control but, going forward, it needs to be controlled or at least measured to assess the importance of this factor in any results.

Although the participants were asked to keep their exercise habits constant during the study, exercise type and frequency during the 4-week intervention differed among the participants. To investigate a possible diet-exercise interaction, the participants were placed into 4 groups based on their self-reported exercise routines (type and frequency) during the 4-week time-restricted eating intervention (Table 3). Group 1 - non-exercisers: participants that exercised $<3$ times per week $(n=6$, Participants = 2, 7, 9, 13, 17, and 19); Group 2 endurance exercisers: participants that endurance trained $\geq 3$ times per week $(n=2$, Participants $=3$ and 4), Group 3 - strength exercisers: participants that strength trained $\geq 3$ times per week $(n=5$, Participants $=3,4,5,10,21$ ), and Group 4 endurance-strength: participants that did similar amounts of endurance and strength training $\geq 3$ times per week ( $n=7$, Participants $=1,11,12,15$, $18,20,22$ ). The strength-training group (Group 3) experienced a significant decrease in fat mass of $0.7 \mathrm{~kg} \pm 1 \mathrm{~kg}$ and no significant lean mass loss following the 4-week intervention (Table 3). No other group had a significant fat mass loss so perhaps there is a diet-exercise training interaction at play. If so, this could be related to increases in fat oxidation as a result of increases in resting metabolic rate (RMR) that is often observed with strength training [10]. In addition, strength trainers eat more protein typically in an attempt to maintain and/or build lean mass making this is another variable, although unclear, that could affect fat mass. Recent research suggests that combining protein with resistance exercise is beneficial for fat free mass preservation during weight loss [11, 12]. Thus, diet and exercise routines need to be controlled in future studies.

Importantly, adherence remained high for all participants (Figure 2), averaging $>5.5 \mathrm{~d} / \mathrm{wk}$ with no significant change, as hypothesized, among weeks suggesting that time-restricted eating is quite well tolerated. In contrast, many other dietary interventions, especially daily energy restriction, are not sustainable over the long term [13]. Thus, timerestricted eating might be a more feasible long-term intervention. Hunger, satisfaction, and fullness from the VVAS, as hypothesized, also remained constant throughout the 4 weeks (Figure 2bcd) suggesting that participants were able to adapt to the new diet routine within the first week, which is quicker than the 2-week adaptation period that has been observed with the alternative day fasting [13]. The maintained level of hunger, satisfaction, and fullness may explain the high adherence level. In the future, daily measures should be taken, at least initially, to determine how many days it takes to adapt to this new eating pattern. Also, prior baseline measures should be obtained to assess how normal eating and time-restricted eating compare.

Of course, the adherence, hunger, satisfaction, and fullness data were self-reported and consequently may be inaccurate. Two strategies were taken to enhance the accuracy of all these measures. The first strategy used was that the participants were informed that reporting accurately was critical for the study. The second strategy was sending weekly motivational emails to the participants that reminded them to record all these measures.

Health concerns with this type of timerestricted eating approach were not expected because the period of fasting was relatively short. No health concerns were observed during this study. However, several individuals experienced mild hunger sensations at the beginning of the study around breakfast time. This appeared to be more psychological than physiological, i.e., participants felt hungry because it was breakfast time not because they needed food [14]. Moreover, this feeling has been shown to disappear after a few days as the body adapts to the new intermittent fasting diet and begins to use stored body fat for energy instead of the food from breakfast [13].

Another important consideration for future studies is the study duration as 4 weeks is a relatively short period of time to observe changes in body composition. Extending studies to 8 or 12 weeks is recommended to assess the significance 
of this novel eating pattern. Moreover, adjusting the time of day and/or the length of the feeding interval would be interesting to investigate. For example, to see the effectiveness of an eating period between 800 to 1600 hours versus 1200 to 2000 hours or whether reducing the fasting period from 16 to 14 or even 12 hours is important.

Finally, recording other measures such as insulin measures, blood glucose, urine ketones, and 24 hour respiratory exchange ratios would help document any physiological effects and changes in fat metabolism resulting from time-restricted eating [15]. Future research should also examine the effect of age, gender, body fat mass, diet, and exercise type on the response to time-restricted eating. Future research should also aim to determine if a daily energy intake decrease results due to the smaller window of eating.

\section{Summary \& Conclusions}

Although there was a significant decrease in body mass there were no significant changes in fat mass following 4-weeks of time-restricted eating (1200 to 2000 hours) diet. However, some individual participants experienced substantial fat loss. Further, strength-trainers seemed to experience greater body fat loss while maintaining their lean mass. Adherence was high and hunger, satisfaction, and fullness remained stable throughout the 4-weeks of time restricted eating indicating that the adaptation to the new eating pattern occurred quickly. Obesity has become a significant public health problem in Canada that is only getting worse [1]. It is a health risk for numerous diseases including mortality, hypertension, type 2 diabetes, coronary heart disease, strokes, cancer, mental illness, and more [2]. With over one fifth of Canadian adults obese and one third overweight, novel interventions are urgently needed [2]. Time-restricted eating appears to be a promising solution; however, more research for longer periods of time, monitoring and controlling exercise and diet, and recording measures such as insulin, blood glucose, urine ketones, and 24-hour respiratory exchange ratios are needed before definitive conclusions can be made. We suggest that time-restricted feeding has potential as a fat loss strategy; however, no definitive conclusions can be made at present.

\section{References}

1. Twells LK, Gregory DM, Reddigan J, Midodzi WK. Current and predicted prevalence of obesity in Canada: a trend analysis. CMAJ Open. 2014; 2(1):18-26. DOI: 10.9778/cmajo.20130016.

2. Centers for Disease Control and Prevention. Adult Obesity Causes \& Consequences. 2015. https://www.cdc.gov/obesity/adult/causes.html

3. Statistics Canada. Overweight and obese adults (selfreported), 2014. 2015. http://www.statcan.gc.ca/pub/82625-x/2015001/article/14185-eng.htm

4. Anderson JW, Knz EC, Frederich RC, Wood CL. Long-term weight-loss maintenance: a meta-analysis of US studies. Am J Clin Nutr. 2001; 74(5):579-584. DOI: http://ajcn.nutrition.org/content/74/5/579.long

5. Tinsley GM, La Bounty PM. Effects of intermittent fasting on body composition and clinical health markers in humans. Nutr Rev. 2015; 73(10):661-674. DOI: 10.1093/nutrit/nuv041.

6. Hatori M, Vollmers C, Zarrinpar A, DiTacchio L, Bushong EA, Gill S, Leblanc M, Chaix A, Joens M, Fitzpatrick JAJ, Ellisman MH, Panda S. Time-Restricted Feeding without Reducing Caloric Intake Prevents Metabolic Diseases in Mice Fed a High-Fat Diet. Cell Metab. 2012; 15(6):848-860. DOI: 10.1016/j.cmet.2012.04.019.

7. Siri WE. Body composition from fluid spaces and density: analysis of methods. 1961. Nutr. 1993; 9(5):480-491.

8. Flint A, Raben A, Blundell JE, Astrup A. Reproducibility, power and validity of visual analogue scales in assessment of appetite sensations in single test meal studies. Int $\mathrm{J}$ Obes Relat Metab Disord. 2000; 24(1):38-48. DOI: 10.1038/sj.ijo.0801083

9. Varady KA, Bhutani S, Church EC, Klempel MC. Short-term modified alternate-day fasting: a novel dietary strategy for weight loss and cardioprotection in obese adults. Am J Clin Nutr. 2009; 90(5):1138-1143. DOI: 10.3945/ajcn.2009.28280.

10. Aristizabal JC, Freidenreich DJ, Volk BM, Kupchak BR, Saenz C, Maresh CM, Kraemer WJ, Volek JS. Effect of resistance training on resting metabolic rate and its estimation by a dual-energy X-ray absorptiometry metabolic map. Eur J Clin Nutr. 2005; 69(7):381. DOI: 10.1038/ejcn.2014.216.

11. Verreijen AM, Engberink MF, Memelink RG, van der Plas SE, Visser M, Weijis PJM. Effect of a high protein diet and/or resistance exercise on the preservation of fat free mass during weight loss in overweight and obese older adults: a randomized controlled trial. Nut J. 2017; 16(1). DOI: http://dx.doi.org.proxy1.lib.uwo.ca/10.1186/s12937017-0229-6.

12. Amamou T, Normandin E, Pouliot J, Dionne IJ, Brochu M, Riesco E. Effect of a high-protein energy-restricted diet combined with resistance training on metabolic profile in older in older individuals with metabolic impairments. J Nutr Health Aging. 2017; 21(1): 67-74. DOI: 10.1007/s12603016-0760-8

13. Klempel MC, Bhutani S, Fitzgibbon M, Freels S, Varady KA. Dietary and physical activity adaptations to alternate day modified fasting: implications for optimal weight loss. Nutr J. 2010; 9(1):1. DOI: 10.1186/1475-2891-9-35.

14. Hams H, Wardle J. The feeling of hunger. BJCP. 2011; 26(2):153-154. DOI: 10.1111/j.2044-8260.1987.tb00745.x

15. Carpenter TM. Tables, Factors, and Formulas for Computing Respiratory Exchange and Biological Transformations of Energy. $4^{\text {th }}$ edition. Washington, DC: Carnegie Institution of Washington; (1921). 